Brazilian Journal

of Chemical

ISSN 0104-6632

Printed in Brazil

Engineering

www.scielo.br/bjce

Vol. 35, No. 02, pp. 745 - 756, April - June, 2018

dx.doi.org/10.1590/0104-6632.20180352s20150727

\title{
SOFT SENSOR MODELS FOR A FRACTIONATION REFORMATE PLANT USING SMALL AND BOOTSTRAPPED DATA SETS
}

\author{
Željka Ujević Andrijić ${ }^{*}$, Matija Cvetnić ${ }^{2}$ and Nenad Bolf ${ }^{1}$ \\ ${ }^{1}$ Department of Measurement and Process Control, Faculty of Chemical Engineering and Technology, \\ University of Zagreb, Savska c. 16/5A, 10000 Zagreb, Croatia, Phone: + (385) (1) 4597-150 E-mail: \\ zujevic@fkit.hr; E-mail: bolf@fkit.hr \\ ${ }^{2}$ Department of Analytical Chemistry, Faculty of Chemical Engineering and Technology, University of \\ Zagreb, Marulićev trg 20, 10000 Zagreb, Croatia, Phone: + (385) (1) 4597-205 E-mail: mcvetnic@fkit.hr
}

(Submitted: November 9, 2015; Revised: November 2, 2016; Accepted: January 3, 2017)

\begin{abstract}
In refinery plants key process variables, like contents of process stream and various fuel properties, need to be continuously monitored using adequate on-line measuring devices. Such measuring devices are often unavailable or malfunction and, hence, laboratory assays which are irregular and time consuming and therefore not suitable for process control are inevitable alternatives. This research shows a comparison of different soft sensor models developed from a small industrial data set with soft sensor models developed from data generated by a bootstrap resampling method. Soft sensors were developed applying multiple linear regression, multivariable adaptive regression splines (MARSpline) and neural networks. The purpose of the developed soft sensors is the assessing of benzene content in light reformate of a fractionation reformate plant. The best results were obtained by the neural network-based model developed on bootstrapped data.
\end{abstract}

Keywords:Bootstrap, neural network, multivariable adaptive regression splines, soft sensor, process modeling.

\section{INTRODUCTION}

Due to growing demands for better product quality but lower product prices and strict safety and environmental rules, there is a need for optimal control of chemical processes. Process control is based on continuously measured process variables in order to get satisfactory product quality with minimum consumption of raw materials and energy. Many of the key process variables which determine product quality in the chemical, petrochemical and oil industry are difficult or even not possible to be continuously measured. With the process knowledge and a lot of easy measurable process variables it is possible to link the secondary, easily measured variables (like the flow, pressure, temperature and level) with variables that are not possible to be continuously measured, the so-called primary variables.

On-line process analyzers are often not available on grounds of malfunction (due to harsh process conditions), during regular maintenance and frequent need for calibration. This problem can be solved by soft sensors application (Zamprogna et al., 2004; Qin, 2007). Soft sensors can work in parallel with analyzers and measuring devices, allowing fault detection schemes.

For the reason of the uncertainty and complexity of industrial processes, fundamental models are often unavailable or inadequate. In industrial plants large quantities of process data are measured and stored

*E-mail address: zujevic@fkit.hr 
in historical data bases which enable identification of data driven models (Fortuna et al., 2007).

In the present study, soft sensors for the prediction of benzene content in the fractionation reformate plant are developed and analyzed. Two linear multiple models, two models of multivariable adaptive regression splines (MARSpline) and two neural network models are developed. Within these models, one model is developed using a small experimental data set, while the other model is developed using bootstrap generated data.

\section{DATA PREPROCESSING}

Selection of the representative process data requires the cooperation of the designer of the soft sensor and plant experts, operators in the control room and process engineers. It is necessary to detect missing data and to remove unwanted components such as outliers, offset, trend and noise.

Since that process analyzers are often inaccessible, the key process properties must be determined by rare and time-consuming laboratory analysis. In such cases, a small number of data is available, so it is necessary to collect as much data as possible during regular operation of the plant. When developing the model with a small number of data there is a strong possibility of poor generalization because the developed model does not take into account process dynamics and all process regimes (Fortuna et al., 2007).

To avoid problems of a small data set several strategies have been considered in the literature. Most of them are based on injecting noise into the available data or by using the bootstrap resampling approach (Napoli and Xibilia, 2011). There is also a method based on an aggregation of neural models, trained on different training data sets, which are obtained by noise injection and bootstrap resampling (Lanuette et al., 1997; Tsai and Der-Chiang, 2008). Injecting noise into the training set means adding zero-mean fixed-variance Gaussian noise, or adding zero-mean Gaussian noise variable variance according to the signal amplitude. Application of ensemble learning algorithms for the improvement of prediction performance of the system can also be found in Caruana et al. (2000), Polikar (2006) and Polikar (2012). Li et al. (2013) applied injection of Gauss noise to the ensemble of the Least Square Support Vector Machine (LS-SVM) model. Data set diversity can be increased by integration of all mentioned techniques, i.e., bootstrap method, noise injection method and stacked neural networks (Di Bella et al., 2007).
Some other models dealing with the small data set problem are presented in literature, like Zhou et al. (2012) who developed a bootstrap aggregated Partial Least Square regression model. Li et al. (2013) applied injection of Gauss noise to the ensemble of the Least Square Support Vector Machine (LS-SVM) model.

A somewhat different approach which deals with dynamic behaviour is considered by Zhu et al. (2009) where an output error method for the identification of a dual fast rate model directly from fast input and slow output data is proposed. A similar approach dealing with dual-rate systems is presented by Ding and Chen (2004), who applied FIR models to predict unmeasurable noise-free outputs, and identify the parameters of underlying fast single-rate models.

In this paper, the bootstrap method is applied with a view to increase training data set diversity and to improve the generalization capabilities of the neural network.

\section{Bootstrap method of generated additional data}

The bootstrap method is a general resampling procedure for estimating the statistical distribution on independent observations introduced in Efron (1979). If there is a function of distribution, $F$, with independent variables, $x_{1}, x_{2}, \ldots x_{n}$, there is a need to investigate the sampling distribution and variability of a function calculated from a sample of size $n$.

The idea of the nonparametric bootstrap method is to simulate data from a cumulative distribution function, $F_{\mathrm{n}} . F_{\mathrm{n}}$ is a discrete probability distribution which gives probability $1 / \mathrm{n}$ for every observed value of $\mathrm{x}_{1}, \mathrm{x}_{2}, \ldots, \mathrm{x}_{\mathrm{n}}$. A sample of size $n$ of a function $F_{\mathrm{n}}$ is a sample of size $n$ drawn with replacement from a set of $x_{1}, x_{2}, \ldots, x_{\mathrm{n}}$. In case of a large sample size, $n$, calculating the distribution is very complicated and therefore it is recommended to create a bootstrap distribution from simple random sampling with replacement.

The basic steps of the bootstrap procedure are (Efron and Tibshirani, 1993):

1. Construction of an empirical probability distribution, $F_{\mathrm{n}}$, from samples by placing a probability of $1 / n$ for each case, $x_{1}, x_{2}, \ldots, x_{n}$ of the sample. This is the empirical distribution function of the samples, which is the nonparametric maximum likelihood estimate of the population distribution, $F$.

2. From the empirical distribution function, $F_{n}$, random samples of size $n$ are drawn with replacement. This procedure is called resampling. 
3. Calculating some statistical parameter, $T_{\mathrm{n}}$ of a resampled sample.

4. Repeating steps 2 and $3, B$ number of times, where $B$ is a large number, in order to create $B$ resamples. Typically, $B$ is at least equal to 1000 when an estimate of the confidence interval around $T_{\mathrm{n}}$ is required.

5. Construction of the relative frequency histogram from the $B$ number of $T_{\mathrm{n}}$ by placing a probability of $1 / B$ for each case. The distribution obtained is the bootstrapped estimate of the sampling distribution of $T_{\mathrm{n}}$.

\section{SOFT SENSOR MODELS}

In the case of long output delays, static models are usually developed. In many industrial processes, where nonlinearity is slightly present and processes are almost steady- state, linear static process can be identified. In processes where defined or undefined nonlinearities are significantly present, nonlinear models must be applied and the identification procedure thus becomes more complex (Fortuna et al., 2007).

\section{Linear multiple model}

The general equation of linear multiple models is presented in Equation (1) where parameters $b_{\mathrm{i}}$ of each input $U_{\mathrm{i}}$ are analogue to the slope, also called the regression coefficients.

$$
y=b_{0}+\sum_{i=1}^{n} b_{i} \cdot U_{i}
$$

$n$ is the number of input variables or predictors.

\section{MARSpline model}

Multivariate Adaptive Regression Splines, or the MARSpline technique has the purpose to predict the value of a set of dependent variables from a set of independent or predictor variables (Friedman, 1991). MARSpline constructs a relationship between dependent and independent variables from a set of coefficients and basis functions that are entirely determined from the data. MARSpline algorithm operates like a multiple piecewise linear regression, where each breakpoint (estimated from the data) defines the "region of application" for a particular (simple) linear equation. The MARSpline algorithm builds models from two-sided truncated functions $(t-x)^{+}$i $(x-$ $t)^{+}$of the predictors (x) which serve as basis functions for a linear or nonlinear expansion that approximate the underlying function $f(x)$. The parameter $t$ is the knot of the basis functions defining the "pieces" of the piecewise linear regression. The "+" sign next to the terms $(t-x)$ and $(x-t)$ denotes that only positive results of the respective equations are considered; otherwise the respective functions evaluate to zero.

Basis functions are defined as:

$$
(x-t)_{+}=\left\{\begin{array}{lc}
x-t & x>t \\
0 & \text { otherwise }
\end{array}\right\}
$$

Parameter $t$ defines the "pieces" of the piecewise linear regression estimated from the data. The MARSpline model for a dependent variable $y$, and $M$ terms can be summarized in the following equation:

$$
y=f(x)=\beta_{0}+\sum_{m=1}^{M} \beta_{m} H_{k m}\left(x_{v(k, m)}\right)
$$

where the summation is over the $M$ terms in the model, and $b_{\mathrm{o}}$ and $b_{\mathrm{m}}$ are parameters of the model. Function $H$ is defined as:

$$
H_{k m}\left(x_{v(k, m)}\right)=\prod_{k-1}^{K} h_{k m}
$$

where $x_{\mathrm{v}}(k, m)$ is the predictor in the $k^{\prime}$ th of the $m^{\prime}$ th product. For the order of interactions $K=1$ the model is additive, and for $K=2$ the model is pairwise interactive.

\section{Neural network}

The full data set was divided randomly into three subsets: the training subset, the testing subset (in order to prevent overfitting) and the validation subset. Weights of the neural network are continually calculated using training data. At the end of each iteration (iteration refers to each passage of all the data for learning through the network), the network predicts a set of values on the test data set. If the test set error is greater than the specified tolerance, the next iteration will be carried out. The process is repeated until the error is less than the specified tolerance, or the predefined number of iteration is reached. To evaluate the model results, a validation data set was used, which is an independent data set that was not applied in training. Multi-Layer Perceptron (MLP) is a typical neural network with a backpropagation algorithm which contains of one input and one output layer, and at least one hidden layer. While the network is in the process of learning, information is propagated back through the network, where the weights are corrected and updated (Nørgaard et al., 2000).

All developed models are evaluated by the comparison of model performance with the performance of the real process using the validation data set within the considered process conditions. It is important to the test model on an independent real 
data set (not used for model estimation) to approve the model applicability and reliability. Validation data set common indicators of model performance are the Pearson correlation coefficient (5) and coefficient of determination (6), defined by the following equations:

$$
\begin{aligned}
R & =\frac{\sum_{i=1}^{n}\left(y_{i}-\bar{y}\right)\left(\widehat{y}_{i}-\overline{\widehat{y}}\right)}{\sqrt{\sum_{i=1}^{n}\left(y_{i}-\bar{y}\right)^{2}\left(\widehat{y_{i}}-\overline{\bar{y}}\right)^{2}}} \\
R^{2} & =\frac{\sum_{i=1}^{n}\left[\widehat{y}_{i}-\bar{y}\right]^{2}}{\sum_{i=1}^{n}\left(y_{i}-\bar{y}\right)^{2}}, 0 \leq R^{2} \leq 1 .
\end{aligned}
$$

The adjusted coefficient of determination, based on the number of degrees of freedom, is given by:

$$
R_{a d j}=1-\frac{n-1}{n-(K+1)} \cdot\left(1-R^{2}\right)
$$

Other popular criteria of numerical model evaluation reported in practical examples are the root mean square error (8) and mean absolute error (9)

$$
\begin{aligned}
& R M S=\sqrt{\frac{\sum_{i=1}^{n}\left(\widehat{y}_{i}-y_{i}\right)^{2}}{n}} \\
& e_{M S E}=\frac{1}{n} \cdot \sum_{i=1}^{n}\left|\widehat{y}-y_{\exp , i}\right|
\end{aligned}
$$

\section{PROCESS DESCRIPTION}

Catalytic reforming is one of the most important processes in the oil industry where refinery crude oil with low octane number in the presence of a catalyst converts into a high octane reformate (Cerić, 2012). Catalytic reformate, as one of the main gasoline components, contains a very high concentration of environmentally undesirable benzene (5-6 vol. \%). In order to satisfy technical and legal norms regarding the benzene content in fuels, currently less than 1 vol. \%, benzene needs to be removed from reformate. European emission standards (such as EURO IV and EURO V) for vehicle exhaust emission and MSAT (Mobile Source Air Toxics) regulations limit the amount of benzene in gasoline, due to the hazardous effect of benzene on health and its negative environmental impact. It is possible to control the formation of benzene by prefractionation of gasoline and by adjustment of the end distillation point of heavy gasoline or by increasing the end distillation point of light gasoline. Unfortunately, it is not possible to completely prevent formation of benzene; therefore, the best solution is the removal of benzene compounds from reformate by post fractionation in a splitter.

Although benzene has a high octane number and high calorific values, its content in light reformate needs to be reduced to $1 \%$. This is due to the fact that benzene is a precursor for the formation of cyclohexane in the process of isomerization, and thus an undesirable component of gasoline (low octane number).

Fractionation of the reformate is used for the separation of light contents found in the reformate. Light reformate contains mostly $\mathrm{C}_{5}$ and $\mathrm{C}_{6}$ hydrocarbons, i.e., the fraction which contains pentane and $\mathrm{C}_{6}$ hydrocarbons with an end distillation point of around $85^{\circ} \mathrm{C}$ at atmospheric pressure. The benzenerich fraction, whose boiling point is between light and heavy reformate, is separated from catalytic reformate.

The fractionation reformate plant with the variables used for soft sensor development is given in Figure 1. Reformate enters into column C-1 where the light reformate is separated from the mixture of heavy reformate and benzene fraction. The bottom product of column C-1 is the feed for the column C-2, where the benzene fraction will be separated from the heavy reformate.

In the laboratory the benzene content is determined and monitored in accordance with the standard EN 238:1996/A1:2003 Liquid petroleum products - Petrol - Determination of the benzene content by infrared spectrometry.

\section{MODEL DEVELOPMENT}

In the refinery the continuous measurement of benzene content in the reformate is crucial. A benzene on-line chromatographic analyzer is frequently under maintenance and sometimes faulty. Considering these facts soft sensors for the continuous on-line estimation of benzene content in light reformate were developed.

During the preliminary test ten input variables (temperatures, pressures and flows) that may affect benzene content in light reformate are considered. Sensitivity analysis, correlation analysis, PCA and PLS methods were performed for the selection of relevant model inputs.

Also, mutual correlations between inputs as well as process engineer experience were taken into account during analysis (Ujević Andrijić et al., 2012).

The desired top product composition is determined by the column top temperature (TC002) controlled by adjusting a pump around flowrate FC-002. Temperature (TC001) and flow (FC001) variation on 


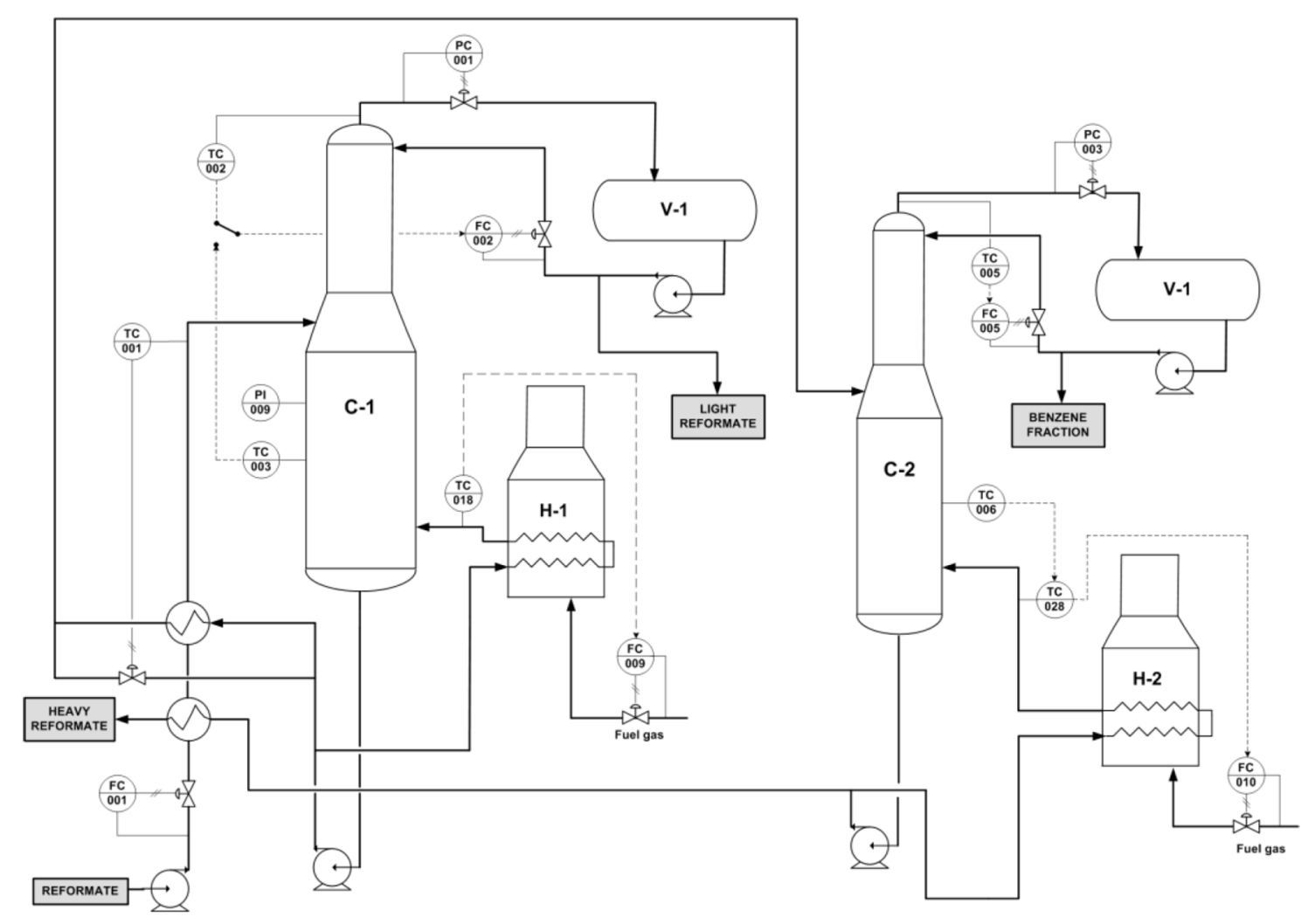

Figure 1. Reformate fractionation plant.

the entrance to the C-1 column (feed) can influence the whole temperature column profile and hence can also influence the product composition. The temperature column profile can also be affected by the outlet temperature of heater H-1 (cascade TC018FC009) which finally has an influence on top product composition. Changes in the temperature profile in the column have an influence on column pressure (PI009). PI009 and TC 003 are also indicators of possible disturbances in the column (fluctuations in top column pressure, temperature or flow).

The following continuously measured variables were chosen as key input variables for particular soft sensor development:

- C1 inlet stream temperature, TC-001 $\left(U_{1}\right)$;

- C1 column bottom temperature (outlet from $\mathrm{H}-1)$, TC-018 $\left(U_{2}\right)$;

- C1 column temperature, TC-003 $\left(U_{3}\right)$;

- C1 column pressure, $\mathrm{PI}-009\left(U_{4}\right)$ and

- Pump around flowrate, FC-002 $\left(U_{5}\right)$.

During the collecting of input and output data the period with different process regime (various process dynamics) is obtained to enable better training. Process data was obtained from the plant history database over a continuous period of three weeks, i.e., 6000 input data with sampling time of five minutes were collected. The model output variable was determined by laboratory measurement every two hours, thus 251 output data were obtained. The number of each input data (sampled every five minutes) must correspond to the number of output data (sampled every two hours); therefore, 251 data of each input and output were synchronized (Figure 2).

Data preprocessing included detecting and removal of outliers and data filtering (Ujević Andrijić et al., 2012). The model development and the model validation used $80 \%$ and 20 percent of the overall data set, respectively. By applying the bootstrap method, from 6000 input samples and 251 output samples, 6000 samples of inputs and 6000 samples of output were generated. Number of data replications, $B$, was set to 1000 . 

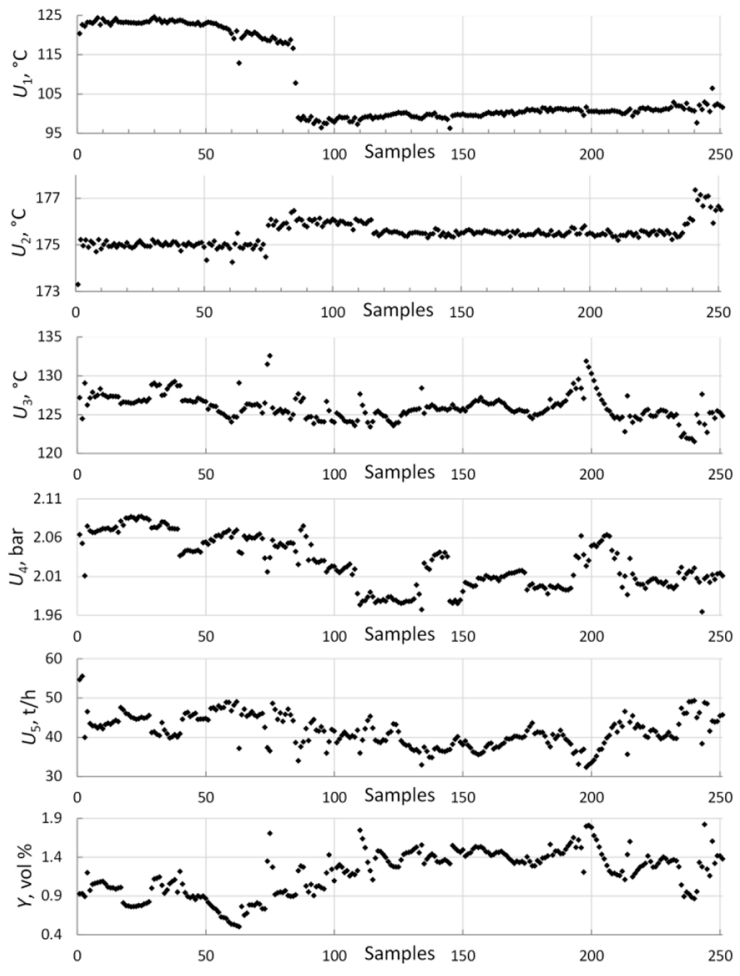

Figure 2. Plot of inputs and output data.

Multiple linear models, MARSpline models and models of MLP neural networks are developed in two ways:

- In the first case, the models were developed from a small data set, containing 201 measured data of every real input and output, while the model is validated on the 50 remaining output data.

- In the second case, 6000 input data generated by the bootstrap method and 6000 generated output data were taken for model development. The model estimation used $80 \%$ randomly chosen data, while $20 \%$, i.e., 1200 , remaining data were used for the model validation. Additional model validation was carried out on 251 laboratory measured output data.

Models were developed in software Statsoft Statistica version 12.5.

\section{RESULTS AND DISCUSSION}

\section{Linear models}

The linear model developed on small data set was presented with the following equation:

$$
\begin{aligned}
& \mathrm{y}=-5.545-0.019 U_{1}+0.012 U_{2}+ \\
& 0.097 U_{3}-2.738 U_{4}+0.004 U_{5}
\end{aligned}
$$

Statistical parameters of the linear model are shown in Table 1. Quite high values of correlation coefficients and small values of absolute and $R M S$ errors indicate good model accuracy.

Table 1. Statistical parameters of the linear model (a small data set).

\begin{tabular}{lcc}
\hline Parameter & Estimation & Validation \\
\hline$R$ & 0.881 & 0.928 \\
$R^{2}$ & 0.777 & 0.862 \\
$R_{\text {adj }}$ & 0.772 & 0.859 \\
$e_{\mathrm{MSE}}$, vol. \% & 0.094 & 0.071 \\
$R M S$, vol. \% & 0.140 & 0.111 \\
\hline
\end{tabular}

Figure 3 shows the graphical comparison between the model output and measured output data using the validation data set. It can be noted that the model output satisfactorily follows the changes in measured output with some minor deviations.

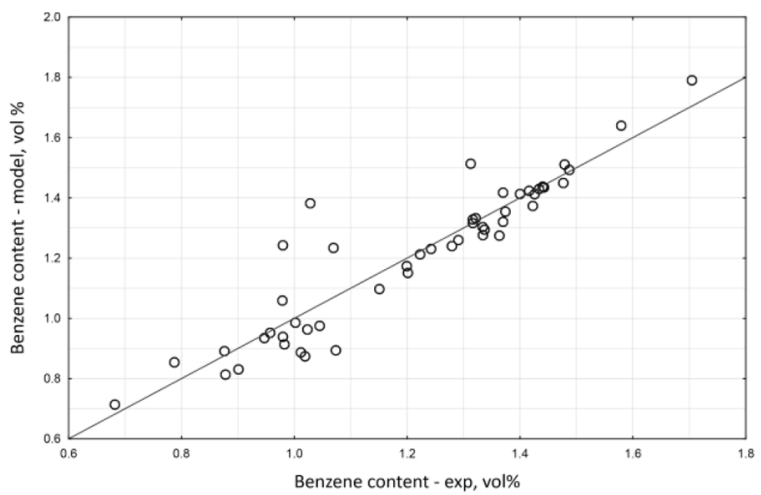

Figure 3. A scatterplot of the linear model vs. validation experimental data (a small data set).

Figure 4 represents the histogram of linear model residuals (differences between estimated and experimental outputs). Residuals are normally distributed with a narrow bell shape, centered on zero. It can be seen that most of the errors lie between -0.1 and 0.1 vol. $\%$ benzene content, which leads to the conclusion that the model satisfactorily matches with laboratory results.

The linear model was developed using 6.000 bootstrapped data (Equation 11).

$$
\begin{aligned}
& \mathrm{y}=-9.644-0.028 U_{1}-0.001 U_{2}+ \\
& 0.094 U_{3}-1.026 U_{4}+0.004 U_{5}
\end{aligned}
$$

Table 2 shows statistical parameters of the linear model developed on the bootstrap expanded data and additionally validated on 251 real output data. From the statistical indicators of model quality in Table 2 it can be concluded that the linear model developed 


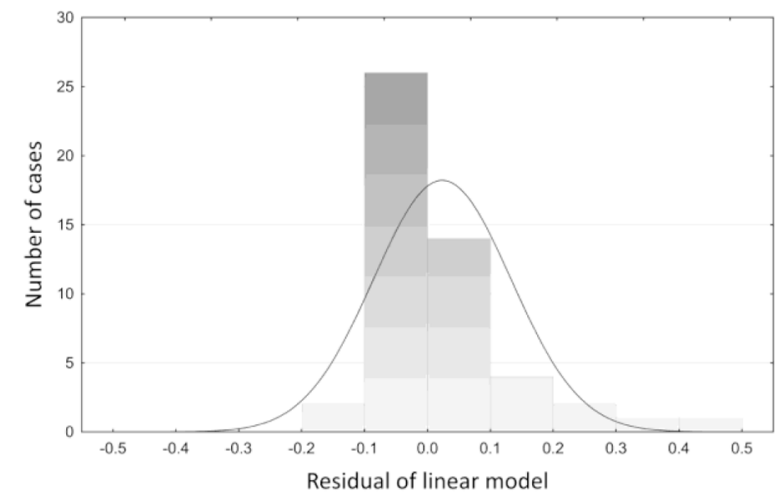

Figure 4. Distribution of the linear model residual on validation data (a small data set).

on bootstrapped data shows somewhat poorer performance than the previously mentioned linear model. The same can be concluded from Figure 5 showing higher dissipation around the $y=x$ direction. Histograms of model residuals (Figure 6) show a wider bell, i.e., the residuals are somewhat higher than the linear model developed on small data. Even though the statistical parameters of the linear model developed on small data set are unexpectedly better, it is not the case with diversity in the results on different data sets. Statistical parameters of the linear model developed on bootstrapped data calculated on all three subsets are very similar, being a good indicator of model applicability to different data sets.

Table 2. Statistical parameters of the linear model (generated data set).

\begin{tabular}{lccc}
\hline Parameter & Estimation & Validation & Real data \\
\hline$R$ & 0.837 & 0.839 & 0.838 \\
$R^{2}$ & 0.700 & 0.704 & 0.702 \\
$R_{a d j}$ & 0.700 & 0.704 & 0.701 \\
$e_{\mathrm{MSE}}$, vol. \% & 0.119 & 0.119 & 0.119 \\
$R M S$, vol. $\%$ & 0.167 & 0.167 & 0.167 \\
\hline
\end{tabular}

\section{MARSpline models}

Several MARSpline models with varying number of basis functions and degrees of interaction using small datasets were developed. The difference in the statistical indicators of these models was insignificant, so the parameters of the simplest developed model were chosen: eight basis functions, one degree of interaction and acriterion penalty of two. In the case of the model developed with bootstrapped data, the model with 13 basis functions, one degree of interaction and criterion penalty of two was selected. Statistical parameters of the MARSpline model developed on the small data set are reported in Table 3. From statistical model

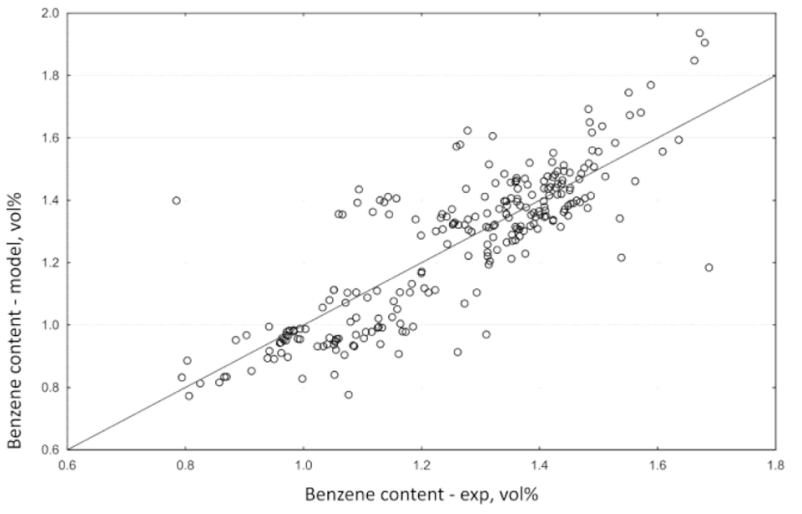

Figure 5. A scatterplot of linear model vs. experimental validation data (generated data set).

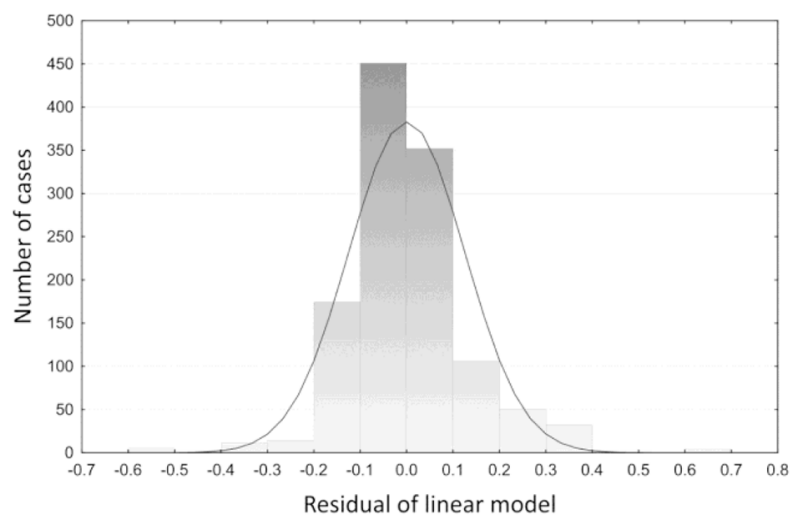

Figure 6. Distribution of linear model residuals on validation data (generated data set).

properties, Figure 7 and Figure 8, good matching of model output with experimental data can be observed.

Statistical parameters of the MARSpline model developed using generated data are presented in Table 4. As in the case of the linear model developed on generated data, the MARSpline model achieved approximately same statistical values in all three subsets, although somewhat better than the linear model. Very good matching of the MARSpline model and experimental data on the validation data set can be seen in Figures 9 and 10.

By comparing models having the same structure types it can be concluded that linear and MARSpline models developed on the generated bootstrapped data have greater ability for generalizations than models developed on small data sets. By comparing the linear models with MARSpline models, it is clear that the MARSpline models have a narrower distribution of model residuals and better graphical comparison of model with experimental data. Statistical parameters of the MARSpline model are also better compared to both linear models; correlation coefficients have higher values while the errors have lower values. 
Table 3. Statistical parameters of the MARSpline model (a small data set).

\begin{tabular}{lcc}
\hline Parameter & Estimation & Validation \\
\hline$R$ & 0.896 & 0.954 \\
$R^{2}$ & 0.803 & 0.911 \\
$R_{\text {adj }}$ & 0.802 & 0.909 \\
$e_{\mathrm{MSE}}$, vol. \% & 0.080 & 0.059 \\
$R M S$, vol. \% & 0.126 & 0.087
\end{tabular}

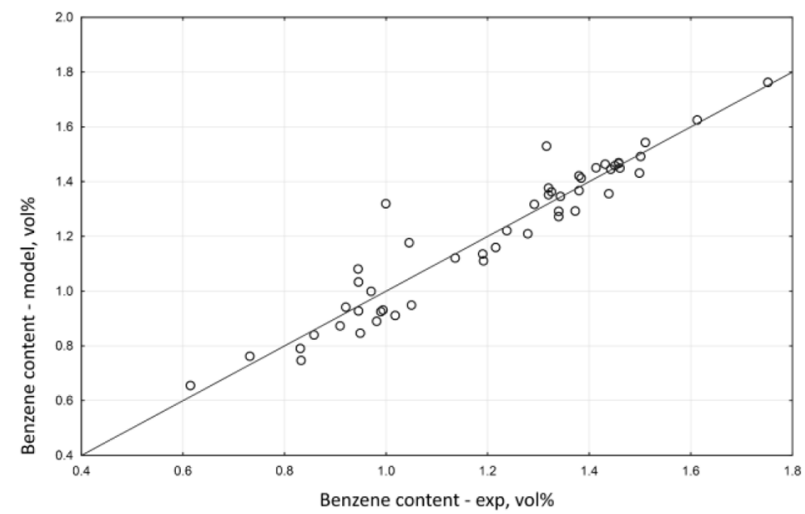

Figure 7. A scatterplot of the MARSpline vs. validation experimental data (a small data set).

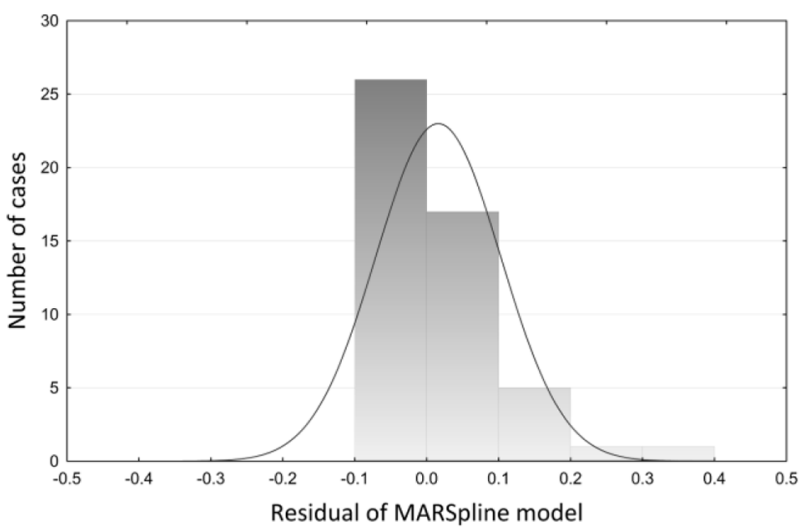

Figure 8. Distribution of MARSpline model residuals on validation data (a small data set).

Table 4. Statistical parameters of MARSpline model (generated data set).

\begin{tabular}{lccc}
\hline Parameter & Estimation & Validation & Real data \\
\hline$R$ & 0.915 & 0.918 & 0.915 \\
$R^{2}$ & 0.838 & 0.843 & 0.836 \\
$R_{a d j}$ & 0.838 & 0.843 & 0.836 \\
$e_{\mathrm{MSE}}$, vol. \% & 0.076 & 0.076 & 0.078 \\
$R M S$, vol. \% & 0.113 & 0.112 & 0.115 \\
\hline
\end{tabular}

\section{Neural network models}

The overall data set is randomly divided into three subsets: a training set which contains $60 \%$ of the data, while the remaining $40 \%$ of data are allocated to the testing $(20 \%)$ and validation sets $(20 \%)$. In order to choose the optimal number of neurons in the

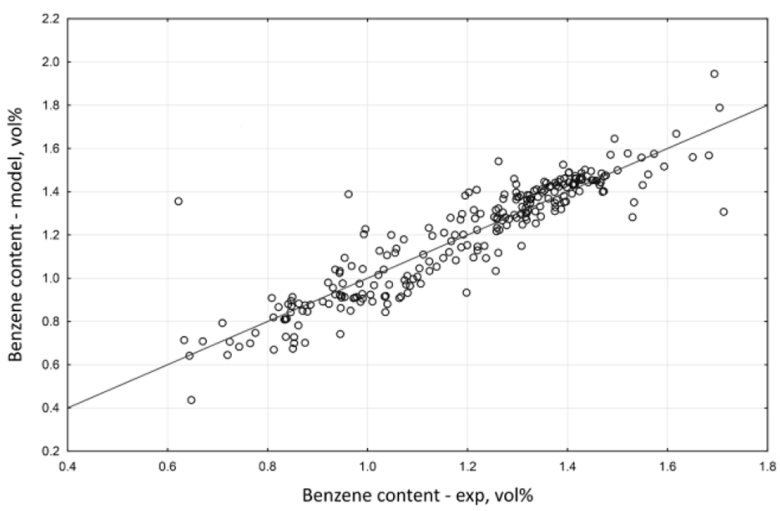

Figure 9. A scatterplot of MARSpline vs. validation experimental data (generated data set).

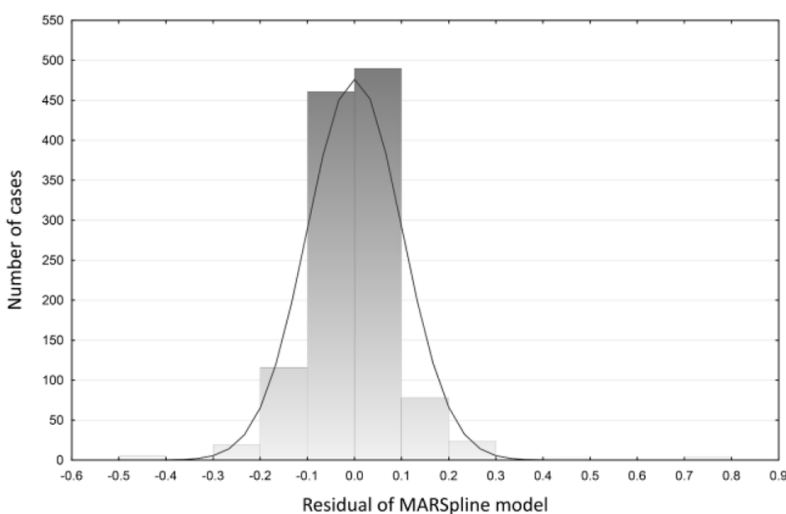

Figure 10. Distribution of MARSpline model residuals on validation data (generated data set).

hidden layer and adequate transfer functions, the five best ones out of 1000 preliminary developed neural networks have been selected. The number of hidden neurons varied from 3 to 8. Exponential, sigmoid, hyperbolic tangent and linear transfer function were tried. In the development of neural network on the basis of the small data set (251 data), the bootstrap subsampling method was used for the selection of learning data. The bootstrap method of subsampling randomly chooses data with a possibility of repeating the same data (i.e., reusing them) an unlimited number of times. It is common that the data set has the same size as the original data set, but with regard to the nature of a method, all data will not be selected. In the preliminary research, using the small data set, the structure of a 5-3-1 network with hyperbolic tangent transfer functions in the hidden layer was chosen. This network contains five neurons in the input layer, three in the hidden and one in the output layer.

In the MLP model developed on 6000 generated data, a 5-8-1structure of the network with logarithmic transfer functions of both layers was chosen. 
Table 5. Selection of the best network developed on the small data set.

\begin{tabular}{lcccccc}
\hline MLP structure & Training Perf. & Test Perf. & Validation Perf. & Training Error & Test Error & Validation Error \\
\hline $5-3-1$ & 0.957 & 0.948 & 0.710 & 0.006 & 0.004 & 0.026 \\
$5-3-1$ & 0.944 & 0.922 & 0.934 & 0.007 & 0.005 & 0.005 \\
$5-3-1$ & 0.909 & 0.857 & 0.941 & 0.011 & 0.011 & 0.005 \\
$5-3-1$ & 0.965 & 0.892 & 0.821 & 0.004 & 0.008 & 0.018 \\
$5-3-1$ & 0.807 & 0.878 & 0.938 & 0.023 & 0.013 & 0.005 \\
\hline
\end{tabular}

There are plenty of possible combinations of the train-test-validation data set regarding random initialization of network weights and random sampling of data to each subset. Therefore, with the aim to improve generalization, 1000 networks of given topology were developed using the small data set, from which five best networks were selected (Table 5). Five best neural networks were chosen using statistical criteria like correlation coefficients and mean square errors of each subset and small diversity in errors of each subset.

Using data generated by the bootstrap method, 1000 networks of given topology were developed from which the five best ones were selected and, among them, the best neural network was chosen, Table 6 .

Statistical parameters of the neural network models developed on the small data set are shown in Table 7. High values of correlation coefficients and small errors point out that the model very well describes the actual data. Such good matching with minor deviations is also observed in Figures 11 and 12.

In Table 8 statistical parameters of the neural network developed on the generated data set are presented. Improved statistical parameters with almost the same values for the estimation and validation data sets show high model accuracy, which is better than the MLP model developed on the small data set. From Figure 13, it can be seen that deviations from the direction $y=x$ are minimal on the entire dataset. In Figure 14 for the histogram, it is clear that most of the errors lie between -0.1 and +0.1 vol. $\%$ of benzene content.

When all developed models are compared, it can be concluded that the best results are achieved by the neural network models developed on generated
Table 7. Statistical parameters of the neural network model (a small data set).

\begin{tabular}{lcc}
\hline Parameter & Estimation & Validation \\
\hline$R$ & 0.932 & 0.934 \\
$R^{2}$ & 0.868 & 0.872 \\
$R_{\text {adj }}$ & 0.867 & 0.869 \\
$e_{\mathrm{MSE}}$, vol. \% & 0.071 & 0.073 \\
$R M S$, vol. \% & 0.101 & 0.104 \\
\hline
\end{tabular}

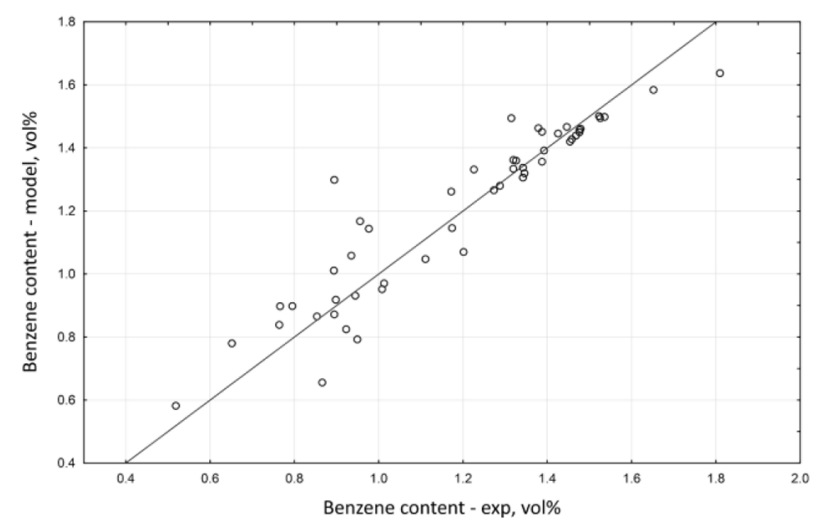

Figure 11. A scatterplot of neural network model vs. validation experimental data (a small data set).

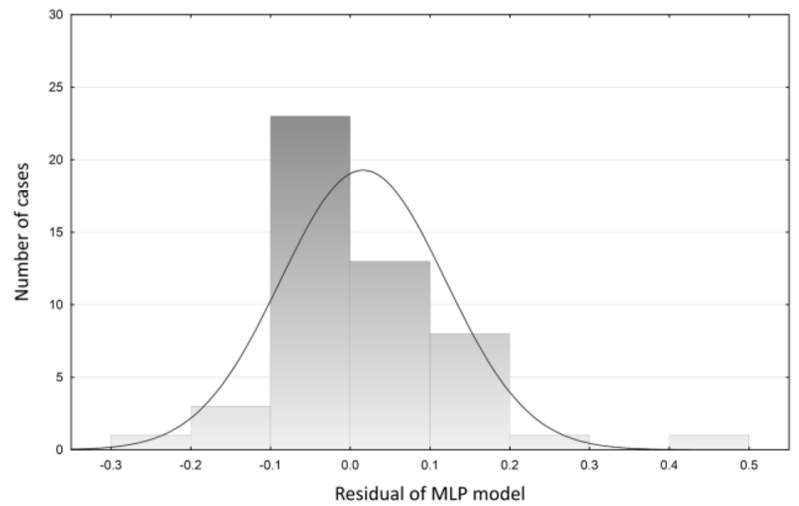

Figure 12. Distribution of MLP model residuals on validation data (a small data set).

Table 6. Selection of the best network developed on the generated data set.

\begin{tabular}{lcccccc}
\hline MLP structure & Training Perf. & Test Perf. & Validation Perf. & Training Error & Test Error & Validation Error \\
\hline $5-8-1$ & 0.976 & 0.971 & 0.974 & 0.002 & 0.002 & 0.002 \\
$5-8-1$ & 0.976 & 0.973 & 0.975 & 0.002 & 0.002 & 0.002 \\
$5-8-1$ & 0.976 & 0.971 & 0.973 & 0.002 & 0.002 & 0.002 \\
$5-8-1$ & 0.962 & 0.953 & 0.959 & 0.003 & 0.003 & 0.003 \\
$5-8-1$ & 0.969 & 0.961 & 0.966 & 0.003 & 0.003 & 0.003 \\
\hline
\end{tabular}


Table 8. Statistical parameters of the neural network model (generated data set).

\begin{tabular}{lccc}
\hline Parameter & Estimation & Validation & Real data \\
\hline$R$ & 0.975 & 0.975 & 0.973 \\
$R^{2}$ & 0.951 & 0.951 & 0.947 \\
$R_{\text {adj }}$ & 0.951 & 0.951 & 0.947 \\
$e_{\mathrm{MSE}}$, vol. \% & 0.044 & 0.011 & 0.002 \\
$R M S$, vol. \% & 0.062 & 0.031 & 0.015 \\
\hline
\end{tabular}

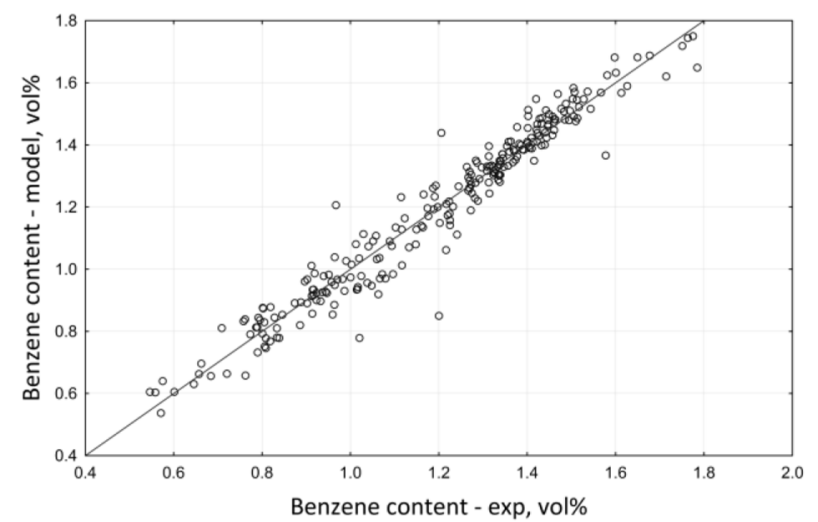

Figure 13. A scatterplot of the neural network model vs. validation experimental data (generated data set).

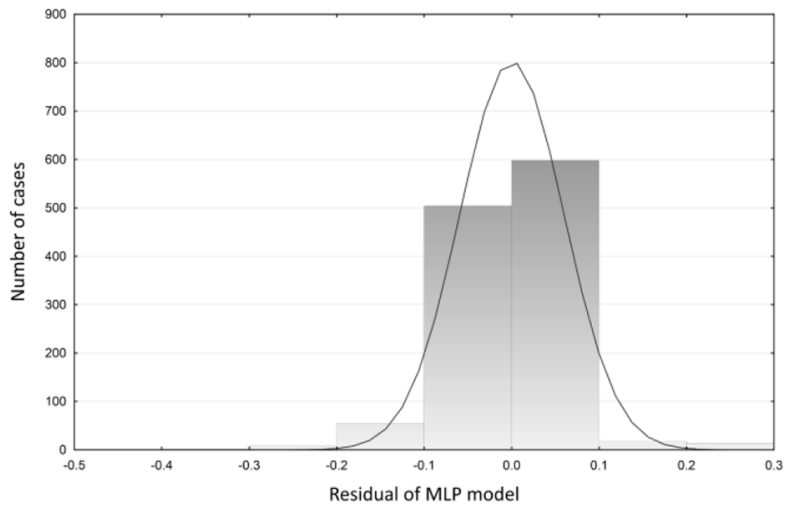

Figure 14. Distribution of MLP model residuals on validation data (generated data set).

data. The presented results are in accordance with the previous similar researches dealing with nonlinear relationships with the limited and small data set problem, using a bootstrapping-based approach (Yuan, 1999; Ivanescu et al., 2006; Tsai et al., 2008; Napoli, 2011).

Residuals of the neural models show narrower distributions of errors than the residuals of linear and MARSpline models.

It is also very important to discern a slight difference in the correlation coefficients between the model applied to training data and to validation data (unlike the linear and MARSpline models developed on small datasets) which, in this case, promises more reliable application of neural network models.

\section{CONCLUSIONS}

This article presented the development and comparison of soft sensor models for the estimation of benzene content in reformate. Models were developed on a small data set as well as on data generated using the bootstrap resampling method. Multiple linear regression models, multivariable adaptive regression spline models and MLP neural network models have been developed.

According to the statistical parameters and diagrams, models developed with neural network achieved the best results, particularly the one developed with generated data.

Multiple linear regression models and MARSpline models gave quite similar and still satisfactory results.

By comparing models developed on the small data set with the ones developed with generated data, it can be observed that the models developed on the small data set show significantly different statistics for the estimation and validation data. It makes the models developed on the small data set less reliable in the comparison with their bootstrapped version.

By additional validation of neural networks models on real-plant data it had been shown that the bootstrap method can be successfully applied to generate additional output data in order to get an improved model performance.

The overall results indicate that the developed soft sensors can be used for continuous analysis of benzene content in reformate at a real plant instead of rare offline laboratory analyses. Finally, the developed soft sensors can be successfully implemented and applied in an advanced process control system.

\section{NOMENCLATURE}

$B$ Number of resamples

$b_{\mathrm{i}}$ Regression coefficients

$\mathrm{e}_{\text {MSE }}$ Mean absolute error

$F$ Function of distribution

FC-002 Pump around flowrate

$F_{\text {n }}$ Discrete probability distribution

$H$ Function defined in Equation (4)

$K$ Order of interactions

LS-SVM Least Square Support Vector Machine model

$M$ Number of terms in Equation (3)

MARSpline Multivariable adaptive regression splines

MLP Multi - Layer Perceptron

MSAT Mobile Source Air Toxics

$n$ Sample size

PCA Principal Component Analysis 
PI-009 Pressure in C1 column

PLS Partial Least Squares

$R$ Pearson correlation coefficient

$R^{2} \quad$ Coefficient of determination

$R_{a d j}$ Adjusted coefficient of determination

$R M S$ Root mean square error

$t$ Knot of the basis functions

TC-001 Inlet stream temperature in $\mathrm{C} 1$ column

TC-003 Temperature in $\mathrm{C} 1$ column

TC-018 Temperature of bottom in C1 column

$T_{\mathrm{n}}$ Some statistical parameter

$U_{\mathrm{i}}$ Inputs

$x_{i}$ Independent variables

$x_{\mathrm{v}}(k, m)$ Predictor in the $k^{\prime}$ th of the $m^{\prime}$ th product

$y$ Dependent variable

Greek Symbols

$\beta$ Parameters of Eq. (3)

\section{REFERENCES}

Caruana, R., Lawrence, S., Giles, L., Overfitting in Neural Nets: Backpropagation, Conjugate Gradient, and Early Stopping, NeuralInformation Processing Systems Conference, NIPS, Denver, 408 (2000)

Cerić, E., Crude Oil; Processes and Products. IBC\&Petrolinvest, Sarajevo (2012).

Di Bella, A., Fortuna, L., Graziani, S., Napoli, G. and Xibilia, M.G., Development of a Soft Sensor for a Thermal Cracking Unit using a small experimental data set, Intelligent Signal Processing, 2007. IEEE. Alcala de Henares (2007).

Efron, B., Bootstrap methods: Another look at the Jackknife, The Annals of Statistics, 7, 1 (1979).

Efron, B. and Tibshirani, R.J., An introduction to the bootstrap. Chapman and Hall, London (1993).

Fortuna, L., Graziani, S., Rizzo, A. and Xibilia, M.G., Soft Sensors for Monitoring and Control of Industrial Processes (Advances in Industrial Control). Springer, London (2007).

Friedman, J.H., Multivariate Adaptive Regression Splines, The Annals of Statistics, 19, 1 (1991).

Ivanescu, V.C., Bertrand, J.W.M., Fransoo, J.C. and Kleijnen, J.P., Bootstrapping to solve the limited data problem in production control: an application in batch process industries, Journal of the Operational Research Society, 57(1) 2-9 (2006)

Lanouette, R., Thibault, J. and Valade, J.L., Process modeling with neural networks using small experimental datasets, Computer and Chemical Engineering, 23(9) 1167-1176 (1997).

Li, Q., Li, X., An, Y. and Ba, W., Soft sensor modeling based on selective ensemble CSLS-SVM algorithm, An International Journal of Research and Surveys, 7(11), 3157 (2013).

Napoli, G. and Xibilia, M.G., Soft Sensor design for a Topping process in the case of small datasets, Computer and Chemical Engineering, 35(11) 24472456 (2011).

Nørgaard, M., Ravn, O., Puolsen, N.K. and Hansen, L.K., Neural Networks for Modelling and Control of Dynamic Systems. Springer, London (2000).

Polikar, R., Ensemble Based Systems in Decision Making. IEEE Circuits and Systems Magazine, 6(3) 21-45 (2006).

Polikar, R., Ensemble Learning, In: Ensemble Machine Learning: Methods and Applications, Springer, New York, NY (2012).

Qin, S.J., Neural networks for intelligent sensors and control-Practical issues and some solutions, Neural Systems for Control, 3 213-234 (1997).

Tsai, T. and Der-Chiang, L., Utilize bootstrap in small data set learning for pilot run modeling of manufacturing systems, Expert Systems with Applications, 35(3) 1293-1300 (2008).

Ujević Andrijić, Ž., Rolich, T. and Bolf, N., Soft Sensor Development for the Estimation of Benzene Content in Catalytic Reformate, Industrial \& Engineering Chemistry Research, 51(7) 3007-3014 (2012).

Yuan, J.L., Bootstrapping nonparametric feature selection algorithms for mining small data sets, Proceedings of the International Joint Conference on Neural Networks. IEEE. Washington, DC (1999).

Zamprogna, E., Barolo, M. and Seborg, D.E., Estimating product composition profiles in batch distillation via partial least squares regression, Control Engineering Practice, 12(7) 917-929 (2004).

Zhou, C., Liu, Q., Huang, D. and Zhang, J., Inferential estimation of kerosene dry point in refineries with varying crudes, Journal of Process Control, 22(6) 1122-1126 (2012).

Zhu, Y., Telkamp, H., Wang, J. and Fu, Q., System identification using slow and irregular output samples, Journal of Process Control, 19(1) 58-67 (2009). 
